\section{Stereotactic and Functional Neurosurgery}

Stereotact Funct Neurosurg 2012;90:379-385 DOI: $\underline{10.1159 / 000339991}$
Received: March 10, 2011

Accepted after revision: June 10, 2012 Published online: August 23, 2012

\title{
Long-Term Outcome of Anterior Thalamic Nucleus Stimulation for Intractable Epilepsy
}

\author{
Kyung Jin Lee ${ }^{a}$ Young Min Shon ${ }^{b}$ Chul Bum Cho c,d \\ Departments of a Neurosurgery and beurology, College of Medicine, Yeouido Saint Mary's Hospital, Catholic \\ University of Korea, Seoul, and 'Department of Neurosurgery, College of Medicine, and d Hallym Institute for \\ Translational Genomics and Bioinformatics, Hallym University Sacred Heart Hospital, Hallym University of Korea, \\ Gyeonggi-do, South Korea
}

\section{Key Words}

Thalamus · Deep brain stimulation · Intractable epilepsy

\begin{abstract}
Background: Many patients with epilepsy have persistent seizures despite treatment with maximal antiepileptic drug therapy and are not candidates for resective brain surgery. Objectives: We investigated the effectiveness of seizure reduction after anterior thalamic nucleus (ATN) stimulation in patients with intractable epilepsy undergoing deep brain stimulation (DBS) of the thalamus. Methods: Patients included in this study had poorly controlled seizures, despite anticonvulsant medication, and were not candidates for surgical resection of an identifiable seizure focus. Fifteen patients with medically refractory epilepsy underwent the placement of bilateral DBS electrodes in the anterior thalamus. The seizure frequency was monitored and compared with the preimplantation baseline. Results: The treatment demonstrated a statistically significant decrease in the seizure frequency, with a mean reduction of $70.4 \%$ (mean follow-up, 27 months). Two of the patients had a remarkable reduction of seizure frequency. Conclusion: It seems to be important that the short-term outcome of ATN DBS reflects the long-
\end{abstract}

term outcome directly. The correlation between the seizure type, characteristics and anticonvulsant effects of ATN DBS did not exhibit significance because of the small number of cases. Therefore, a longer-term follow-up with a larger group of patients is required to fully evaluate the safety and effectiveness of this treatment modality.

Copyright $\odot 2012$ S. Karger AG, Basel

\section{Introduction}

Despite the maximal level of antiepileptic drug therapy, as many as $50 \%$ of patients with epilepsy have persistent seizures. Approximately one third of epileptic patients who have suffered from refractory seizures are not candidates for resective surgery because of the involvement of the eloquent areas or the multifocal nature of the ictal onset $[1,2]$.

The concept of direct brain stimulation for epilepsy was first introduced by Cooper et al. [3] in the 1970s. There have been many direct brain stimulations on various brain subcortical targets for epilepsy after their first attempt. Typical subcortical targets for epilepsy are the cerebellum [3-5], anterior thalamic nucleus (ATN) [6-9],

\section{KARGER}

Fax +41613061234 E-Mail karger@karger.ch www.karger.com
(C) 2012 S. Karger AG, Basel

$1011-6125 / 12 / 0906-0379 \$ 38.00 / 0$

Accessible online at:

www.karger.com/sfn
Chul Bum Cho

Department of Neurosurgery, Hallym University Sacred Heart Hospita

896, Pyeongchon-dong, Dongan-gu, Anyang-si

Gyeonggi-do 431-070 (South Korea)

Tel. +82 31380 3044, E-Mail medics0919@gmail.com 
Fig. 1. Diagram demonstrating the simulation of the planned trajectory to the ATN for DBS using a surgical navigation system (Medtronic Stealth navigation; Medtronic) with a superimposed standard stereotactic atlas.

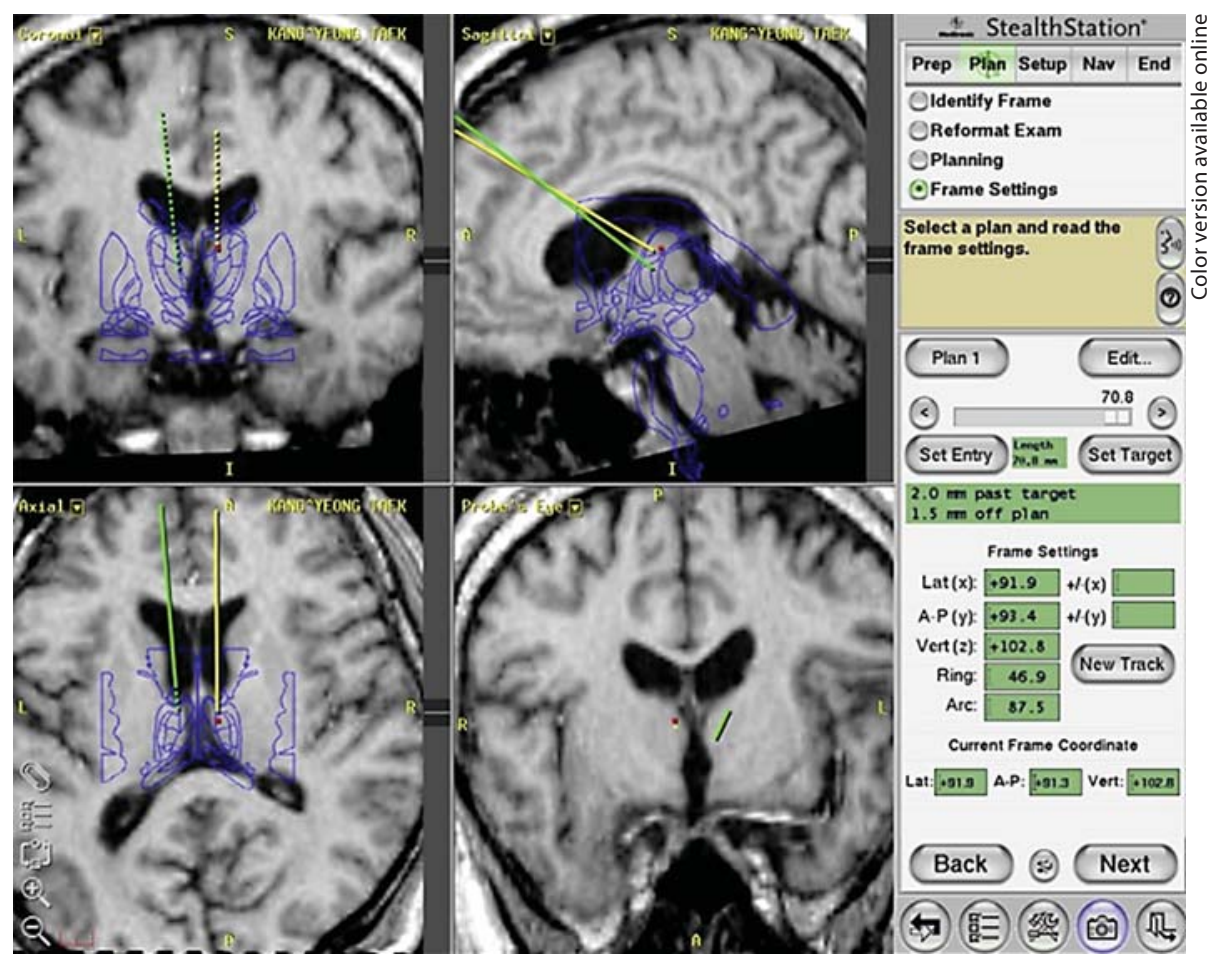

subthalamic nucleus $[10,11]$, caudate nucleus [12], centromedian nucleus [13-15] and the mesial temporal structures [16-18].

It is a recent trend to perform this attempt through deep brain stimulation (DBS). Since DBS has been shown to be effective and safe in the treatment of movement disorders, its application to the treatment of intractable seizures has been extended farther.

Until now, stimulation of the ATN is often suggested as an ideal target. The SANTE study was a multicenter, randomized, double-blind parallel-group study of $110 \mathrm{im}$ planted patients with refractory partial or secondarily generalized seizures [19]. During the blinded evaluation period, patients with the stimulation on had a seizure reduction of $38 \%$ as compared to $14.5 \%$ with the stimulation off.

ATN DBS has emerged as an effective and acceptable alternative therapy for patients with intractable seizures. Despite its reported efficacy in several studies, the precise mechanism of its antiepileptic action remains elusive and requires to be established, and information regarding the long-term outcome of ATN DBS in patients with pharmacoresistant epilepsy is limited.

Therefore, the purpose of this study was to evaluate the long-term outcome of ATN DBS for intractable epilepsy compared with the short-term outcome.

\section{Materials and Methods}

\section{Patient Selection}

Patients included in this study had poorly controlled seizures (more than 4 per month), despite multiple anticonvulsant drugs or previous seizure operation, and were not candidates for surgical resection of an identifiable seizure focus. All patients had undergone a previous scalp video-EEG monitoring to characterize the seizure types and localization. The study was approved by the institutional review board of the Catholic University of Korea, and informed written consent was obtained from all patients or their family members.

\section{Operative Technique}

The procedure for the electrode implantation began with placement of the stereotactic frame under local anesthesia. After attachment of the magnetic resonance imaging (MRI) localizer, an MRI was obtained with both fast spin echo inversion recovery and standard $\mathrm{T}_{2}$-weighted images. According to a standard stereotactic atlas, the ATN is $5 \mathrm{~mm}$ lateral and $12 \mathrm{~mm}$ superior to the midcommissural point [20]. Direct visualization of the ATN is also possible by coronal and axial MRI. We used a surgical navigation system (Medtronic Stealth navigation; Medtronic, Minneapolis, Minn., USA) to perform simulation of the planned trajectory from the entry point to the ATN and to confirm avoidance of sulcal vessels (fig. 1). Surgery was performed under local anesthesia. The patient was placed in a supine position and the head of the bed was elevated $30^{\circ}$. Two burr holes were placed, and the dura and pia were incised and cauterized. A guide cannula was inserted and advanced deep into the brain to a point $10 \mathrm{~mm}$ from 

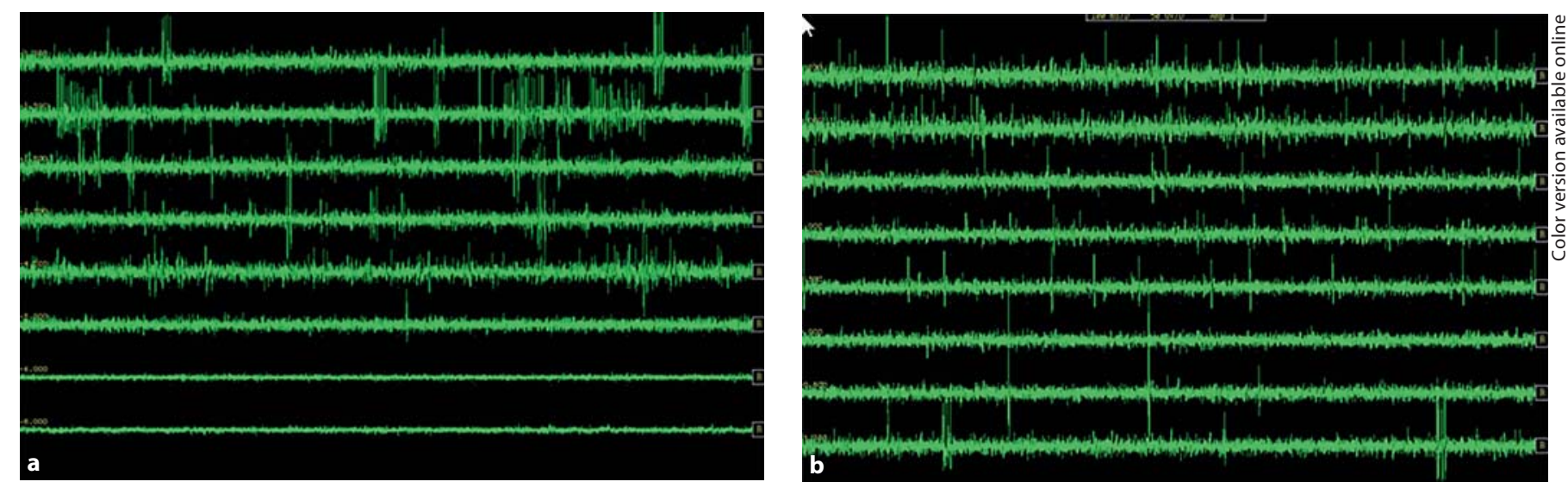

Fig. 2. a, b Recordings from a single-unit monopolar electrode shown at various depth levels, identifying the entry into the thalamic tissue after traversing the lateral ventricle.

Fig. 3. a Axial $\mathrm{T}_{2}$-weighted brain MRI of patient 1 demonstrating the localization of stimulation electrodes after implantation. b Coronal $\mathrm{T}_{2}$-weighted brain MRI of patient 1 demonstrating the localization of stimulation electrodes in the ATN.
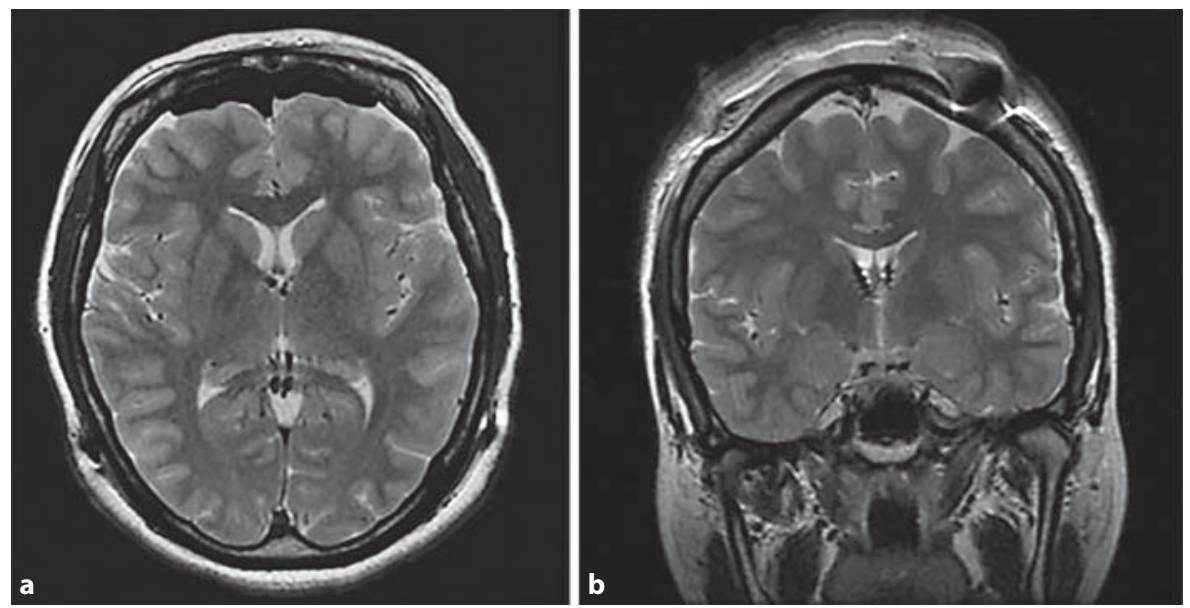

the desired target under direct fluoroscopic and Leksell frame guidance. Microelectrode recordings were done to identify the neural signals obtained from the ATN and dorsal medial nucleus (fig. 2). We used Medtronic 3387 leads in the ATN. In terms of lead contacts, the bottom one and 2 contacts were in the dorsal medial nucleus, and the upper 2 and 3 in the ATN because the height of the ATN is about $6 \mathrm{~mm}$ in humans and the quadripolar electrode span is $10.5 \mathrm{~mm}$ [9]. After the implantation of electrodes at the ATN, the EEG recording during the insertion of electrodes with or without external electrical stimulation (Medtronic 7432 External Programmer, Medtronic) was performed to determine whether a driving response could be elicited (slow frequency 5-10 $\mathrm{Hz}$, intensity 3-7 V, pulse width $90 \mathrm{~ms}$ ). The programmable pulse generators (Medtronic 7424 ITREL II Pulse Generator, Medtronic) were surgically placed under general anesthesia in a subcutaneous pocket in the subclavicular region and connected to the stimulation leads by means of a lead extension (Medtronic 7495 Lead Extension, Medtronic), which was tunneled under the skin of the neck and scalp. The placement location of the lead was confirmed with a cranial magnetic resonance image (fig. 3).
For the chronic ATN high-frequency stimulation, our stimulation protocols of ATN DBS are as follows: frequency 100-185 $\mathrm{Hz}$, voltage $1.5-3.1 \mathrm{~V}$, pulse duration $90-150 \mu \mathrm{s}$, continuous stimulating mode, and in 1 or 2 stimulated DBS electrode contacts. Activation and programming of the implanted pulse generator started 1 week after implantation.

The stimulation parameter was adjusted for a satisfactory clinical response, and determined individually for each patient.

\section{Clinical Assessment}

The monthly seizure frequency data was obtained during the follow-up visits on the 3rd, 6th, 12th, 18th, 24th, 36th and 48th months, or more after implantation of the ATN DBS from parents and caregivers. The preoperative seizure frequency was collected for a 3-month baseline period before the DBS implantation. This collected seizure frequency divided by 3 was defined as the baseline seizure frequency. The seizure frequency before and after the ATN DBS was noted according to the specific seizure type (atonic, partial complex, generalized tonic-clonic, absence and myoclonic). Antiepileptic drugs were typically left unchanged during 
Fig. 4. The seizure reduction percentage compared to the preoperative state.

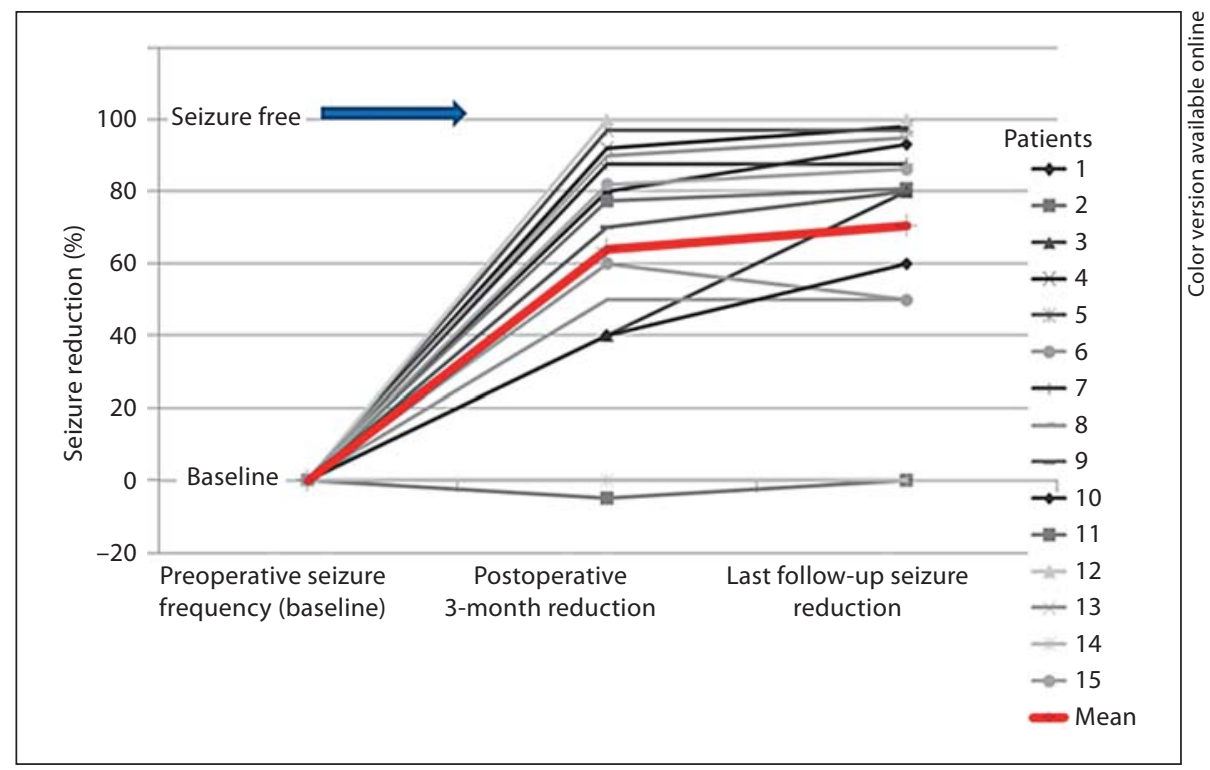

the first 12 months unless adjustments were necessitated by increasing seizures or drug toxicity. Thereafter, medication adjustment was permitted. During periods of medication adjustment, we did not change any stimulation parameters in order to evaluate the effect of medication changes alone. The following outcome variables were assessed: (a) total seizure frequency at postoperative month three relative to baseline, (b) total seizure- frequency between postoperative month three and the last follow up. The statistical significance of changes in seizure frequency was measured with the Wilcoxon signed-rank test (SPSS, version 12, Chicago, Ill., USA). The statistical significance between the seizure etiology and the seizure reduction percentage and between the age and seizure reduction percentage were measured with the Mann-Whitney U test and Spearman's correlation coefficient, respectively. Results were considered statistically significant when $\mathrm{p} \leq 0.05$.

\section{Results}

The total number of ATN DBS in intractable patients during the period between December 16, 2004, and December 31, 2009, was 15. The follow-up included 15 patients (9 males and 6 females). The median follow-up period was $39.00 \pm 14.33$ months (range, 24-67). The median age of the patient at the time of the operation (ATN DBS) was $30.53 \pm 12.18$ years (range, $14-54$ years). The median seizure onset age was $13.53 \pm 5.59$ years (range, 7-29). The median seizure duration from the first seizure attack to the operation (ATN DBS) was $17.00 \pm 11.10$ years (range, 3-39). Clinical features for each of the 15 patients in this study are shown in table 1 .
Preoperative, postoperative month 3 and the last follow-up seizure frequency per month are $120.33 \pm 304.10$ (range, 2-1,200), $32.07 \pm 70.84$ (range, 0-270) and 27.67 $\pm 61.23(0-230)$, respectively. The mean seizure reduction percentage between the preoperative and last followup seizure frequency is $70.51 \pm 32.09 \%$ and the mean seizure intensity at the last follow-up was $0.60 \pm 0.73$ (table 2). The seizure reduction percentages compared with the preoperative state (baseline) are shown in figure 4.

The seizure frequency per month at postoperative month 3 and the last follow-up period after the ATN DBS shows a significant reduction in the frequency of seizures (Wilcoxon signed-rank test, $\mathrm{p}=0.002, \mathrm{p}=0.001$, respectively). The reduction percentage of the seizure frequency at postoperative month 3 has a significant relationship with the reduction percentage of the seizure frequency during the last follow-up period (Wilcoxon signed-rank test, $\mathrm{p}=0.032$ ). The last follow-up seizure reduction percentage was also stratified according to 4 factors: age at seizure onset, age at the time of ATN DBS, etiology and seizure duration from age at seizure onset to age at the time of ATN DBS. There was no significant factor associated with the seizure reduction percentage including: age at seizure onset (Spearman's correlation, $\mathrm{r}=0.013, \mathrm{p}=$ 0.96), age at the time of ATN DBS (Spearman's correlation, $r=0.294, p=0.28$ ), etiology (Mann-Whitney U test, $\mathrm{p}=0.206$ ) and seizure duration from the age of seizure onset to the recorded age at the time of the ATN DBS (Spearman's correlation, $\mathrm{r}=0.244, \mathrm{p}=0.38$ ). 

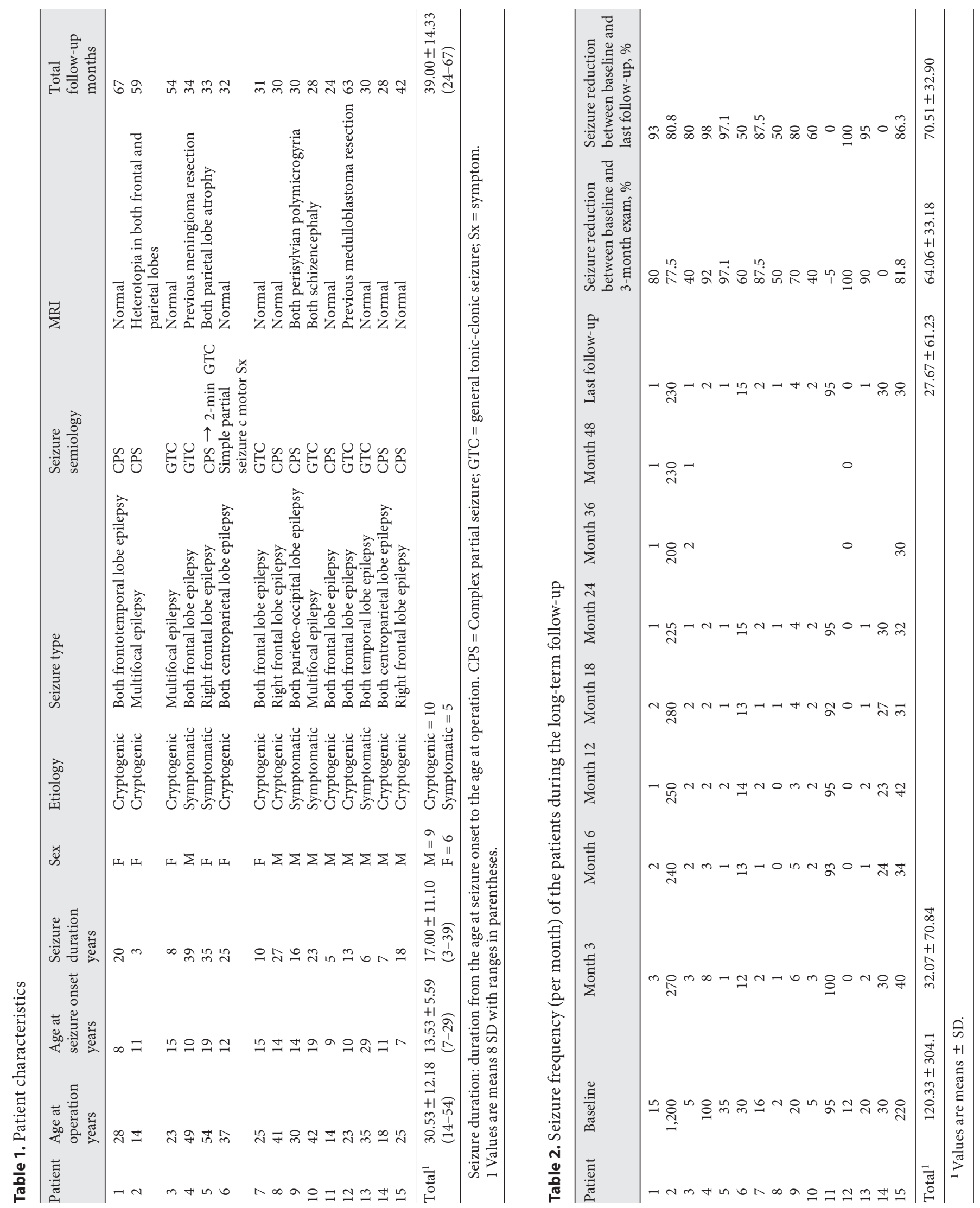
There was no major complication observed such as intracranial hemorrhage. However, we had one complication during the removal of the DBS devices from the wound infection, including one of a single patient who had demonstrated a great improvement in the seizure frequency.

\section{Discussion}

We had previous knowledge regarding the potential limitations of resective and disconnection surgery for epilepsy and recognized the success of vagal nerve stimulation for epilepsy in the 1990s and 2000s. In addition, we have accumulated experience in the performance of DBS for the treatment of Parkinson's disease and maintain the confidence that the advantages of DBS include reversibility, titrability and safety. This background has encouraged members of the neurosurgical field to recently conduct the DBS procedure for epilepsy.

There are two underlying neurophysiological concepts regarding the use of brain stimulation for epilepsy [21]. The first is that brain stimulation in the epileptogenic zone alters the neurophysiological properties of the tissue and can have an inhibitory effect. The second is the alteration of epileptic networks by stimulation of crucial network nodes. The ATN DBS involves the second concept. The ATN which is within the reticulothalamocortical system is part of the circuit of Papez and alters the thalamocortical output [22].

The advantages of targeting the ATN are as follows. First, the ATN is well defined and includes a relatively large nucleus for stereotaxy. Second, the microelectrical recording of structures which the electrode passes through are typical (ventricle, ATN, nucleus dorsomedialis in order). Third, the synchronization and desynchronization on the EEG is typical of both the low- and high-frequency stimulation of the ATN, respectively. Fourth, the possibility of stimulation of both the ATN and nucleus dorsomedialis (which is also involved in the signal propagation in the epileptogenic circuit). Fifth, there are rare stimulation-induced symptoms in contrast to the vagal nerve stimulation-induced laryngeal symptoms. Therefore, it is easy to attempt a double-blind study of the ATN DBS and rule out the placebo effect. According to Schaltenbrand and Wahren [20], the ATN is composed of the nucleus anteroprincipalis thalami, nucleus anterodorsalis thalami and nucleus anteromedialis thalami. The diameter of the ATN is approximately $6 \mathrm{~mm}$ suggesting the placement of the stimulation electrode of the ATN on the nucleus anteroprincipalis thalami. Evidence to support our statement is as follows. First, the majority of the ATN includes the nucleus anteroprincipalis thalami. The second is that in the case of the stimulation of both the ATN and the nucleus dorsomedialis, the stimulation electrode is probably located on the nucleus anteroprincipalis thalami due to electrode trajectory. The third is that the targeting of the nucleus anterodorsalis thalami is very difficult because its diameter is very small. The fourth is that the targeting of the nucleus anteromedialis thalami needs additional posterior entry points compared with Kocher's point because it is located on a more inferior part than the nucleus anteroprincipalis.

To our knowledge, this is a rare report regarding the long-term outcome of the ATN. The mean follow-up period for our patients is 39 months and so it is possible to evaluate the clinical significance of both the short- and long-term outcomes. We considered the time point for the short-term evaluation as 3 months following the operation, because we thought that 3 months after the operation is the most ideal time for ruling out the effects of microthalamotomy and the adjustment of the stimulation parameter as well. According to our results, the short-term outcome directly reflects the long-term outcome specifically, as the remarkable seizure reduction appeared rapidly in the short-term period and this effect remained through the long term. This result suggests that the ATN DBS is effective in the long as well as the short term and that we can predict the long-term outcome by the evaluation of the short-term one. The age at the time of the operation, the age at seizure onset, seizure duration (from seizure onset age to age at operation) and etiology have no clinical significance in relation to the observed seizure reduction percentage. We think that a possible reason may be the small case number included in the study.

Several patients experienced effects that were not predicted. In patient 1 , the seizure attacks only occurred when she was extremely tired. Patient 3 exhibited a generalized tonic-clonic type seizure which changed to a focal type seizure limited to the head. Patient 8 was able to predict the onset of upcoming seizure attacks. Patient 10 displayed alterations in her sexual libido including increased orgasms. Patient 13 demonstrated a decrease in the frequency of seizure attacks; however, the seizures continued when he was extremely intoxicated. Patient 15 exhibited a decrease in the frequency of seizures when the targeting area was changed from the subthalamic nucleus to the ATN. The mechanism of this unpredictable out- 
come is not clear. However, we hypothesize that its mechanism may be closely related with the limbic structure as the ATN is a component within the limbic structure networks. These unpredictable outcomes deserve further consideration regarding the mechanism of ATN DBS for epilepsy and the extended utility of DBS for the treatment of various diseases.

It is essential that the short-term outcome of the ATN DBS directly reflects the long-term outcome. The correlation between the seizure type, characteristics and anticonvulsant effect of the ATN DBS did not demonstrate significance because of the small case numbers. Therefore, an extended long-term follow-up with a larger group of patients is required to fully evaluate the safety and effectiveness of this treatment modality.

\section{Acknowledgement}

This research was supported by Basic Science Research Program through the National Research Foundation of Korea funded by the Ministry of Education, Science and Technology (grant No. 2011-0013232).

\section{References}

1 Kwan P, Brodie M: Early identification of refractory epilepsy. N Engl J Med 2000;342: 314-319.

-2 Lesser R: Unexpected places. Neurology 1999;52:1117-1118.

-3 Cooper I, Amin I, Riklan M, Waltz J, Poon T: Chronic cerebellar stimulation in epilepsy: clinical and anatomical studies. Arch Neurol 1976;33:559-570

4 Wright G, McLellan D, Brice J: A doubleblind trial of chronic cerebellar stimulation in twelve patients with severe epilepsy. J Neurol Neurosurg Psychiatry 1984;47:769774.

5 Van Buren J, Wood J, Oakley J, Hambrecht F: Preliminary evaluation of cerebellar stimulation by double-blind stimulation and biological criteria in the treatment of epilepsy. J Neurosurg 1978;48:407-416.

-6 Kerrigan JF, Litt B, Fisher RS, Cranstoun S, French JA, Blum DE, Dichter M, Shetter A, Baltuch G, Jaggi J, Krone S, Brodie M, Rise M, Graves N: Electrical stimulation of the anterior nucleus of the thalamus for the treatment of intractable epilepsy. Epilepsia 2004;45:346-354.

7 Lee K, Jang K, Shon Y: Chronic deep brain stimulation of subthalamic and anterior thalamic nuclei for controlling refractory partial epilepsy. Acta Neurochir Suppl 2006;99: 87-91.
8 Lim S, Lee S, Tsai Y, Chen IA, Tu P, Chen J, Chang $\mathrm{H}, \mathrm{Su} \mathrm{Y}$, Wu T: Long-term anterior thalamus stimulation for intractable epilepsy. Chang Gung Med J 2008;31:287-296.

-9 Hodaie M, Wennberg RA, Dostrovsky JO, Lozano AM: Chronic anterior thalamus stimulation for intractable epilepsy. Epilepsia 2002;43:603-608

10 Benabid A, Minotti L, Koudsie A, de Saint Martin A, Hirsch E: Antiepileptic effect of high-frequency stimulation of the subthalamic nucleus (corpus luysi) in a case of medically intractable epilepsy caused by focal dysplasia: a 30-month follow-up. Technical case report. Neurosurgery 2002;50:1385-1391.

11 Charbades S, Kahane P, Minotti L, Koudsie A, Hirsh E, Benabid A: Deep brain stimulation in epilepsy with particular reference to the subthalamic nucleus. Epileptic Disord 2002;4:83-93.

12 Chkhenkeli S, Chkhenkeli I: Effects of therapeutic stimulation of nucleus caudatus on epileptic electrical activity of brain in patients with intractable epilepsy. Stereotact Funct Neurosurg 1997;69:221-224.

13 Velasco F, Velasco M, Velasco A, Jimenez F, Marquez I, Rise M: Electrical stimulation of the centromedian thalamic nucleus in control of seizures: Long-term studies. Epilepsia 2005;36:63-71.

-14 Velasco M, Velasco F, Velasco A, Brito F, Jimenez F, Marquez I, Rojas B: Electrocortical and behavioral responses produced by acute electrical stimulation of the human centromedian thalamic nucleus. Electroencephalogr Clin Neurophysiol 1997;102:461-471.
15 Velasco M, Velasco F, Velasco A: Centromedian-thalamic and hippocampal electrical stimulation for the control of intractable epileptic seizures. J Clin Neurophysiol 2001;18: 495-513.

16 Tellez-Zenteno J, McLachlan R, Parrent A, Kubu C, Wiebe S: Hippocampal electrical stimulation in mesial temporal lobe epilepsy. Neurology 2006;66:1490-1494.

17 Boon P, De Herdt V, Vonck K, Van Roost D: Clinical experience with vagus nerve stimulation and deep brain stimulation in epilepsy. Acta Neurochir Suppl 2007;97:273-280.

18 Velasco A, Velasco F, Velasco M, Trejo D, Castro G, Carrillo-Ruiz J: Electrical stimulation of the hippocampal epileptic foci for seizure control: a double-blind, long-term follow-up study. Epilepsia 2007;48:1895-1903.

19 Fisher RS: Release of the Stimulation of the Anterior Nucleus of the Thalamus in Epilepsy (SANTE) trial results. American Epilepsy Society meeting. Seattle, 2008.

20 Schaltenbrand GHR, Wahren W: Atlas for Stereotaxy of the Human Brain, ed 2. Stuttgart, Thieme, 1977

21 Jobst B: Brain stimulation for surgical epilepsy. Epilepsy Res 2010;89:154-161.

22 Mirski M, Rossell L, Terry J, Fisher R: Anticonvulsant effect of anterior thalamic high frequency electrical stimulation in the rat. Epilepsy Res 1997;28:89-100. 